\title{
Motor Dysfunction in Olivopontocerebellar Atrophy Is Related to Cerebral Metabolic Rate Studied with Positron Emission Tomography
}

\author{
Guy Rosenthal, BA, ${ }^{*}$ Sid Gilman, MD,* Robert A. Koeppe, PhD,† Karen J. Kluin, MS, \\ Dorene S. Markel, MS, Larry Junck, MD, ${ }^{*}$ and Stephen S. Gebarski, MD\$
}

\begin{abstract}
We compared the severity of motor dysfunction with local cerebral metabolic rates for glucose (ICMRGIc) and the degree of tissue atrophy in 30 patients with olivopontocerebellar atrophy (OPCA). We devised a scale to quantitate the degree of ataxia in the neurological examinations. ICMRGIc was measured with ${ }^{18} \mathbf{F}$-2-fluoro-2-deoxy-D-glucose and positron emission tomography (PET). Tissue atrophy was assessed by visual rating of computed tomographic scans. PET studies revealed marked hypometabolism in the cerebellar vermis, cerebellar hemispheres, and brainstem of OPCA patients compared with 30 control subjects. A significant correlation was found between severity of motor impairment and ICMRGlc within the cerebellar vermis, both cerebellar hemispheres, and the brainstem. $A$ significant but weaker relationship was noted between the degree of tissue atrophy in these regions and clinical severity. Partial correlation analysis revealed that motor dysfunction in OPCA correlated more strongly with ICMRGlc than with the amount of tissue atrophy. These results suggest that the clinical manifestations of OPCA are more closely related to the metabolic state of the tissue than to the structural changes in the cerebellum.
\end{abstract}

Rosenthal G, Gilman S, Koeppe RA, Kluin KJ, Markel DS, Junck L, Gebarski SS. Motor dysfunction in olivopontocerebellar atrophy is related to cerebral metabolic rate studied with positron emission tomography. Ann Neurol 1988;24:414-419

Olivopontocerebellar atrophy (OPCA) is a progressive neurological disorder involving neuronal degeneration in the cerebellar cortex, pons, and inferior olivary nuclei [1-4]. The disease often displays autosomal dominant inheritance, though autosomal recessive transmission and sporadic cases occur as well. Early clinical features of the disease include a disorder of gait and dysarthria. Later, impairment of coordinated movements of all the limbs and further impairment of the bulbar musculature develop. The rate of progression of the signs and symptoms associated with OPCA varies widely between patients.

The diagnosis of OPCA is usually based on clinical evaluation, since most laboratory investigations show no abnormalities. Cerebrospinal fluid protein may be slightly elevated, and the electroencephalogram may show an excessive amount of slow activity [5]. Auditory evoked potentials are sometimes abnormal [6], and impaired sensory nerve conduction has been reported $[7,8]$. The results of immunological and biochemical investigations are usually normal, though a deficiency of glutamate dehydrogenase has been reported in the tissue fibroblasts and platelets of some individuals with autosomal recessive and autosomal dominant forms of the disorder [9-16].

The list of conditions that can lead to cerebellar dysfunction in adults is extensive and includes malformations, degenerations, vascular diseases, infections, neoplasms, remote effects of neoplasms, toxic/metabolic disorders, and demyelinating disease. The diagnosis of OPCA is usually suggested by the presence of atrophy of the cerebellum and brainstem on computed tomographic (CT) scanning, often in association with abnormalities of brainstem auditory evoked responses and peripheral nerve studies. Because none of these tests is diagnostic for OPCA, an extensive evaluation is needed to rule out the many other possibilities of progressive cerebellar dysfunction in an adult.

Recently, we used positron emission tomography (PET) and ${ }^{18}$ F-2-fluoro-2-deoxy-D-glucose ( ${ }^{18}$ F-FDG)
From the Departments of *Neurology, Internal Medicine (Division of Nuclear Medicine), $\longleftarrow$ Physical Medicine and Rehabilitation, and $\$$ Radiology, The University of Michigan, Ann Arbor, MI 48109.

Received Jan 12, 1988, and in revised form Apr 5. Accepted for publication Apr 5, 1988.
Address correspondence to Dr Gilman, The University of Michigan, Department of Neurology, 1914/0316 Taubman Health Care Center, 1500 E Medical Center Dr, Ann Arbor, MI 48109-(0)316. 
to study local cerebral metabolic rates of glucose (lCMRGlc) in 30 patients with OPCA and 30 normal volunteers with the goal of defining a metabolic pattern that might aid in the diagnosis of OPCA [17]. We found a significant decline in ICMRGlc in the cerebellar vermis, both cerebellar hemispheres, and the brainstem of patients with OPCA when compared to agematched control subjects. In $80 \%$ of OPCA patients, ICMRGlc values normalized to the cerebral cortex were more than two standard deviations below the mean of the normal group in at least one cerebellar region or the brainstem. The thalamus and the cerebral cortex were not hypometabolic. Moreover, we have shown that most OPCA patients studied have some degree of tissue atrophy detected by CT, though in some patients the atrophy is only mild [17]. Determination of the specificity of these PET and CT findings awaits studies of patients with other diseases affecting the cerebellum.

In the current study we sought to define the relationship between the severity of the clinical manifestations in OPCA and the metabolic rate of the cerebellum and brainstem. We also intended to explore the relationship between observed clinical severity and tissue atrophy as assessed by CT. Moreover, we aimed to determine whether the clinical signs of the disease are more closely linked to a decline in ICMRGlc or to the amount of tissue atrophy.

\section{Methods}

We studied 30 individuals with OPCA and 30 normal control subjects. The studies were approved by the institutional review board and informed consent was obtained from all subjects. The normal control subjects had no history of neurological disease and no significant abnormalities on neurological or general physical examination. The subjects were taking no medication known to affect central nervous system (CNS) function or to cause CNS side effects. All subjects were carefully screened for alcoholism, and none with a clear history of alcoholism were admitted into the study. The patients with OPCA included 13 men and 17 women with OPCA with an average duration of illness of 6 \pm 5 years and a range of 1 to 22 years. Sixteen cases were of sporadic occurrence, while 14 patients presented with a family history of similar illness in first-degree relatives. Among these, all but 1 had a history compatible with autosomal dominant transmission. One had a history compatible with autosomal recessive transmission. The diagnosis of OPCA was made on the basis of the history, physical examination, neurological examination, laboratory tests to exclude other diseases, and the findings on CT scans. None of the patients had disorders of sensory function adequate to cause ataxia of movement. The laboratory tests included complete blood counts; serum profiles of hepatic and renal function; brainstem auditory, visual, and somatosensory evoked potentials; serum levels of vitamins $E, B_{12}$, and folic acid; and studies of thyroid function. A search was made for an occult malignancy and included a pelvic examination in the women, a
Table 1. Somatic Motor Signs

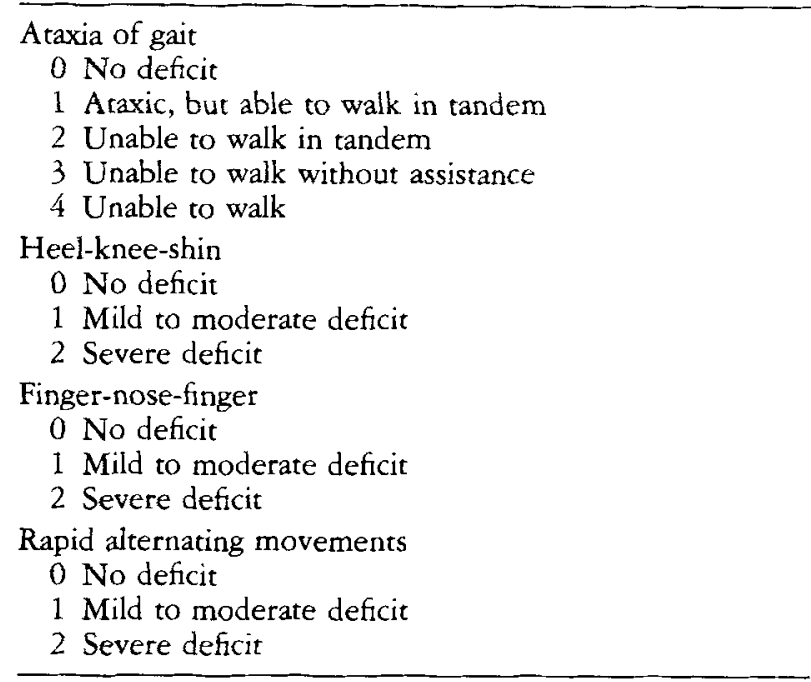

Table 2. Cranial Motor Signs

Nystagmus
0 Not present
1 Present
Ocular dysmetria
0 Not present
1 Present
Ataxia of speech
0 No perceptible deficit
1 Mild deficit
2 Moderate deficit
3 Severe deficit
Impaired tongue movements
0 Not present
1 Present

prostate examination in the men, acid phosphatase levels, stool guaiac tests for occult blood, and chest roentgenograms.

To quantitate clinical severity in OPCA, we devised a rating scale (Tables 1,2). The signs chosen for inclusion in the scale were selected because of their importance in the diagnosis of OPCA and in determining the extent of progression of the disease. We relied on our clinical experience with this disorder to formulate the scale. The scale was devised before any analysis of PET or CT data.

Four somatic motor signs accounted for 10 of the 16 potential points in our scale and four cranial motor signs accounted for six points. This was intended to reflect the relative importance of somatic motor and cranial motor deficit in the clinical progression of the disease process. Of the somatic motor signs, two are associated with the lower extremities and two with the upper extremities. Of the cranial motor signs, two each are associated with the mouth/larynx and eyes. The number of points associated with each individual sign represents its importance in determining clinical severity in OPCA in our clinical judgment. Clinical ratings were applied to each patient by two of the authors (G. R. and S. G.). 
The scoring of ataxia of speech was based on an evaluation conducted by one of the authors (K. J. K.). The rating system used for assessment of speech has been described previously [18].

All subjects were studied in the University of Michigan Division of Nuclear Medicine P.E.T. Facility while lying supine, awake, and blindfolded in a quiet room. Each subject was placed in a head holder that maintained the head immobile throughout the study. The head was aligned along the orbitomeatal line with a laser. Scans were performed 30 to 75 minutes after the injection of ${ }^{18} \mathrm{~F}$-FDG. Radiochemical purity of ${ }^{18} \mathrm{~F}-\mathrm{FDG}$, synthesized by a modification of the method described by Ehrenkaufer and colleagues [19], was greater than $95 \%$. Five to $10 \mathrm{mCi}$ were injected intravenously, and PET scans were performed with a TCC PCT $4600 \mathrm{~A}$ tomograph with an in-plane resolution of $11 \mathrm{~mm}$ full width at half maximum (FWHM) and a $\mathrm{Z}$-axis resolution of $9.5 \mathrm{~mm}$ FWHM. Five planes with 11.5 -mm center-to-center separation were imaged simultaneously. Four sets of scans were taken per patient, including two interleaved sets through lower brain levels and two interleaved sets through higher brain levels for a total of 20 slices, each separated by $5.75 \mathrm{~mm}$. An attenuation correction was calculated by the standard ellipse method, modified to account for attenuation from the head holder and skull.

Blood samples were collected from the radial artery. ICMRGlc was calculated using a three-compartment model and single scan approximation described by Phelps and coworkers $[20]$ with gray matter kinetic constants derived from normal subjects [21]. As described fully in a previous article [17], regions of interest (ROIs) were studied in the cerebellar hemispheres, cerebellar vermis, brainstem, thalamus, and cerebral cortex. Data were collected from the ROIs by placing a $22 \times 11 \mathrm{~mm}$ parallelogram over each cerebellar hemisphere, an $11 \times 18 \mathrm{~mm}$ rectangle over the cerebellar vermis, an $11 \times 15 \mathrm{~mm}$ rectangle over the brainstem, and an $11 \times$ $11 \mathrm{~mm}$ square over each thalamus. Each ROI was centered over a local peak in ICMRGlc. (For reference, an individual image element [pixel] is $3.75 \mathrm{~mm} \times 3.75 \mathrm{~mm}$ in size.) Data were obtained from two slices containing the cerebellum and brainstem and from one slice containing the thalamus. ROIs from the cerebellar vermis were posterior to the fourth ventricle. The brainstem ROI chiefly reflects the pons, but the mesencephalon or medulla oblongata could be partially represented. Data from the cerebral cortex were obtained by measuring ICMRGlc in the cortical ribbon from consecutive slices beginning with the lowest slice containing the basal ganglia and continuing until the mesial cortex became visible. This was accomplished with a computerized routine that enhances the contrast in the images, then selects a 15 -mm-wide band extending inward from the cortical rim. The mean metabolic rate was computed for each of these ROIs. The metabolic rate for the subcortical regions studied was normalized to the metabolic rate of the entire cerebral cortex.

CT scans were obtained in OPCA patients with a GE 9800 tomograph. The scans were read by a neuroradiologist (S. S. G.) who was informed only of the patient's age and diagnosis. The neuroradiologist was unaware of the patient's clinical history or findings on PET study. A rating scale previously described was used to quantitate the degree of atrophy in each cerebellar hemisphere, the cerebellar vermis, and the brainstem [17]. In this rating scale, the neuroradiologist took into account the degree of atrophy expected on the basis of the patient's age. Representative CT scans have been published [17].

Spearman rank correlations were used to compare metabolic and CT data to clinical scores and to assess the relationship between CT rating and ICMRGlc. The partial correlations method was used to eliminate the effect of the relationship between ICMRGlc and tissue atrophy when these two variables were measured against clinical score.

\section{Results}

The neurological findings in the OPCA patients are summarized in Table 3. All the patients presented with a disorder of gait and an ataxic dysarthria. Some impairment of coordinated limb movements was noted in all patients. Nystagmus was present in 20 patients and ocular dysmetria in 8. Seventeen patients demonstrated impaired tongue movements. Limb spasticity, hyperreflexia, and extensor plantar responses were present in 9, 6, and 8 patients, respectively.

We found a significant relationship between the overall severity of the clinical signs and a decline in ICMRGlc normalized to the cerebral cortex for the cerebellar vermis, left and right cerebellar hernispheres, and the brainstem of OPCA patients (Table 4). No correlation between ICMRGlc and clinical score was noted for the thalamus. As an example, Figure 1 portrays the relationship between clinical score and metabolic rate in the cerebellar hemispheres.

We also found a statistically significant relationship between clinical severity and the amount of tissue atrophy assessed by CT in the cerebellar vermis and both cerebellar hemispheres (Table 5). The correlations were of a slightly lesser magnitude than those noted between ICMRGIc and clinical score. A significant correlation was not observed between brainstem atrophy rating and clinical score.

The amount of tissue atrophy determined by CT correlates significantly with the decline in ICMRGlc measured by PET in each of the three cerebellar regions studied and the brainstem (cerebellar vermis, $r$ $=-0.47, p<0.005$; left cerebellar hemisphere, $r=$ $-0.45, p<0.01$; right cerebellar hemisphere, $r=$ $-0.54, p<0.005$; brainstem, $r=0.38, p<0.05$ ). Figure 2 demonstrates the relationship between $C T$ rating and metabolic rate in the cerebellar hemispheres. Because PET and CT measures were correlated with each other, we used the partial correlations method to assess the relationship of these two variables to clinical score, independent of the effect of the other variable. Partial correlations reveal that when the effect of the relationship between ICMRGlc and tissue atrophy is eliminated, the amount of tissue atrophy no longer correlates significantly with clinical severity in OPCA. That is, at any given metabolic rate, the amount of tissue atrophy in the cerebellar and 
Table 3. Clinical Features in OPCA Patients Studied

\begin{tabular}{|c|c|c|c|c|c|c|c|c|c|c|c|c|c|c|c|}
\hline $\begin{array}{l}\text { Patient } \\
\text { No. }\end{array}$ & Sex & $\begin{array}{l}\text { Family } \\
\text { History } \\
\end{array}$ & Age & $\begin{array}{l}\text { Duration } \\
\text { (yr) }\end{array}$ & Gait ${ }^{*}$ & $\mathrm{HKS}^{\mathrm{b}}$ & $\mathrm{FNF}^{b}$ & $\mathrm{RAM}^{\mathrm{b}}$ & $\begin{array}{l}\text { Ataxic } \\
\text { Speech }\end{array}$ & $\begin{array}{l}\text { Nystag- } \\
\text { mus }^{\mathrm{j}}\end{array}$ & $\begin{array}{l}\text { Ocular } \\
\text { Dysmetria }^{d}\end{array}$ & $\begin{array}{l}\text { Tongue } \\
\text { Disorder }\end{array}$ & $\begin{array}{l}\text { Spas- } \\
\text { ticity }\end{array}$ & $\begin{array}{l}\text { Hyper- } \\
\text { reflexia }\end{array}$ & $\begin{array}{l}\text { Extensor } \\
\text { Plantar }^{d}\end{array}$ \\
\hline 1 & M & Spor & 30 & 16 & ++ & ++ & ++ & $t+$ & +++ & + & + & + & - & - & - \\
\hline 2 & $\mathrm{M}$ & Spor & 38 & 9 & +++ & + & - & + & + & + & - & + & + & + & + \\
\hline 3 & F & Dom & 54 & 6 & ++ & + & + & ++ & $+t$ & + & + & - & - & - & + \\
\hline 4 & $\mathrm{~F}$ & Dom & 71 & 1 & +++ & + & - & - & + & - & - & - & + & - & - \\
\hline 5 & F & Dom & 44 & 5 & +++ & + & + & + & + & + & - & - & - & - & - \\
\hline 6 & $\mathrm{~F}$ & Spor & 53 & 6 & ++ & + & - & + & ++ & - & - & + & - & - & - \\
\hline 7 & M & Spor & 60 & 3 & +++ & $+t$ & ++ & ++ & ++ & + & + & - & - & - & - \\
\hline 8 & F & Dom & 28 & 5 & $+t$ & ++ & + & + & + & + & - & - & + & + & - \\
\hline 9 & $\mathrm{~F}$ & Spor & 59 & 2 & $+++t$ & ++ & $+t$ & $t+$ & $++t$ & + & - & + & - & - & + \\
\hline 10 & $\mathrm{~F}$ & Spor & 65 & 6 & ++++ & + & + & ++ & + & - & - & - & - & - & - \\
\hline 11 & M & Dom & 68 & I & ++ & + & + & + & ++ & + & - & - & + & + & - \\
\hline 12 & $\mathrm{~F}$ & $\operatorname{Rec}$ & 41 & 7 & + & + & + & + & ++ & + & - & + & + & - & + \\
\hline 13 & $\mathrm{~F}$ & Spor & 59 & 2 & ++++ & + & t & $t+$ & ++ & + & + & + & + & - & + \\
\hline 14 & $\mathrm{~F}$ & Dom & 45 & 6 & ++ & ++ & + & + & + & + & + & -- & - & - & - \\
\hline 15 & M & Spor & 50 & 5 & $t+$ & + & + & + & +++ & - & + & - & -- & - & - \\
\hline 16 & F & Dom & 39 & 3 & $+t$ & + & ++ & ++ & + & + & - & - & - & - & - \\
\hline 17 & $\mathrm{~F}$ & Spor & 52 & 4 & $+t$ & + & + & ++ & ++ & - & - & + & - & - & - \\
\hline 18 & M & Spor & 57 & 20 & ++ & + & + & + & ++ & + & - & + & - & - & -- \\
\hline 19 & $M$ & Dom & 48 & 5 & + & + & + & + & ++ & - & - & + & + & - & - \\
\hline 20 & $E$ & Spor & 71 & 3 & +++ & + & + & ++ & ++ & + & - & - & - & - & - \\
\hline 21 & $\mathrm{~F}$ & Dom & 61 & 11 & $+t$ & + & + & + & + & - & - & + & - & + & - \\
\hline 22 & $\mathrm{~F}$ & Dom & 65 & 15 & +++ & + & ++ & $+t$ & ++ & + & - & + & - & + & + \\
\hline 23 & $M$ & Spor & 65 & 2 & $++t$ & + & + & $+t$ & + & - & - & + & - & - & - \\
\hline 24 & $\mathrm{~F}$ & Dom & 25 & 7 & ++++ & ++ & ++ & ++ & +++ & - & - & + & - & - & - \\
\hline 25 & $\mathrm{~F}$ & Spor & 66 & 3 & $+t$ & + & + & + & ++ & + & - & - & - & - & + \\
\hline 26 & M & Spor & 68 & 4 & ++ & + & t & + & +++ & + & + & + & - & - & - \\
\hline 27 & $M$ & Spor & 69 & 3 & $+t$ & + & + & + & + & + & - & + & - & - & - \\
\hline 28 & $M$ & Spor & 55 & 4 & +++ & ++ & $+t$ & ++ & +++ & + & - & + & + & + & + \\
\hline 29 & M & Dom & 47 & 22 & ++++ & ++ & ++ & ++ & $t+t$ & - & - & + & + & - & - \\
\hline 30 & M & Dom & 38 & 2 & ++ & + & + & + & + & + & + & - & - & - & - \\
\hline
\end{tabular}

${ }^{2}$ Gait: $-=$ no deficit; $+=$ mildly ataxic $++=$ ataxic and unable to walk in tandem; $+++=$ unable to walk without assistance; $++++=$ unable to walk. bHKS, FNF, RAM: $-=$ no deficit; $+=$ mild to moderate defict; $++=$ severe deficit.

Ataxic speech: $-=$ no deficit; $+=$ mild deficit $++=$ moderate deficit $+++=$ severe deficit

'Nystagmus, ocular dysmetria, tongue disorder, spasticity, hyperreflexia, extensor plantar reflex: - = not present; $+=$ present.

HKS = heel-knee-shin test; FNF = finger-nose-finger test; $\mathrm{RAM}=$ rapid alternating movements; Spor $=$ sporadic; Dom = dominantly inherited; Rec = recessively inherited.

Table 4. Correlations Between Clinical Impairment Score and ICMRGli (Normalized) ${ }^{\mathrm{a}}$

\begin{tabular}{lc}
\hline Brain Region & Correlation Coefficient \\
\hline Cerebellar vermis & $-0.53^{\mathrm{b}}$ \\
Left cerebellar hemisphere & $-0.53^{\mathrm{b}}$ \\
Right cerebellar hemisphere & $-0.48^{\mathrm{b}}$ \\
Brainstem & $-0.39^{\mathrm{c}}$ \\
Thalamus & 0.02 \\
\hline
\end{tabular}

aSpearman rank correlation coefficients of the relationship berween the clinical impairment score and the local cerebral metabolic rates for glucose (ICMRGIc), normalized to the metabolic rate of the entire cerebral cortex. Clinical impairment score was determined from the neurological examination and perceptual analysis of speech. $\mathrm{b}_{p}<0.005$

sp $<0.05$.

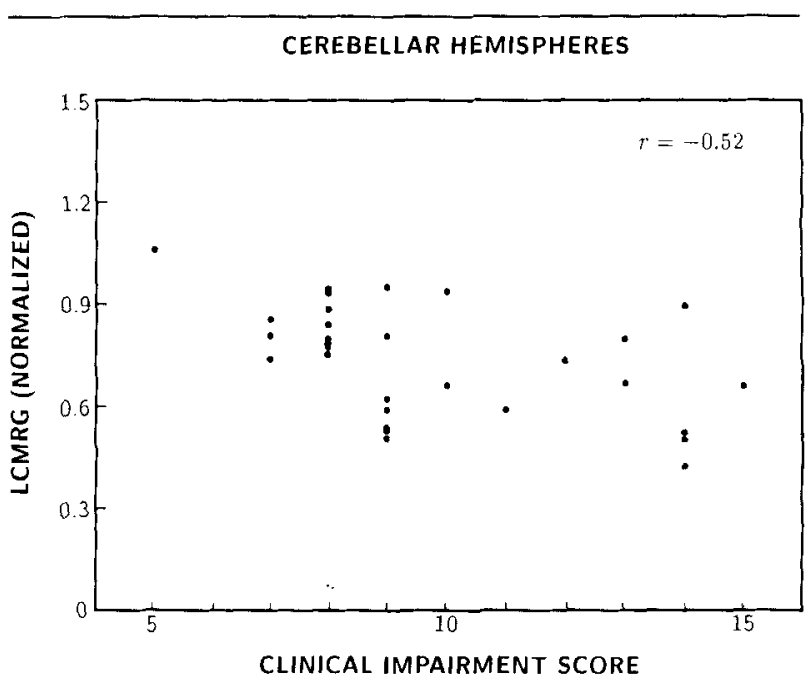

Fig 1. Severity of dinical impairment plotted against the mean local cerebral metabolic rate for glucose ( $L C M R G)$ normalized to the cerebral cortex in the left and right cerebellar bemispheres. $A$ significant relationship $(\mathrm{p}<0.005)$ is seen between clinical severity and $L C M R G$. 
Table 5. Correlations Between Clinical Impairment Score and Tissue Atropby

\begin{tabular}{lc}
\hline Brain Region & Correlation Coefficient \\
\hline Cerebellar vermis & $0.42^{\mathrm{b}}$ \\
Left cerebellar hemisphere & $0.36^{\mathrm{b}}$ \\
Right cerebellar hemisphere & $0.42^{\mathrm{b}}$ \\
Brainstem & -0.04 \\
\hline
\end{tabular}

${ }^{2}$ Spearman rank correlation coefficients. Clinical impairment score was determined from the neurological examination and perceptual analysis of speech. Tissue atrophy was determined by visual rating of computed tomographic scans. $\mathrm{b}_{p}<0.05$.

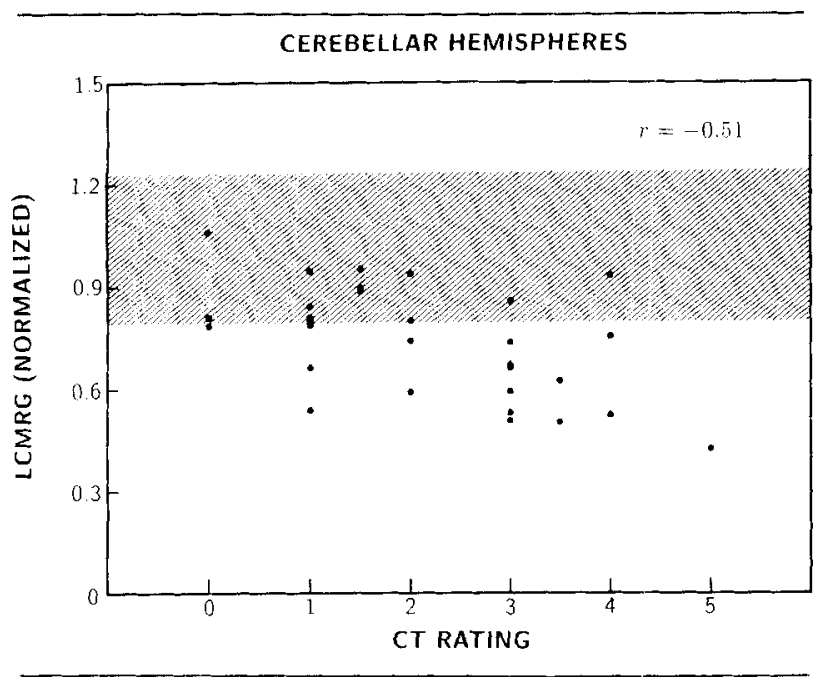

Fig 2. Graph of the mean local cerebral metabolic rate for glucose (LCMRG), normalized to the cerebral cortex in the left and right cerebellar bemispheres, plotted against the degree of atrophy observed in computed tomographic (CT) scans. A significant relationship $(\mathrm{p}<0.005)$ is seen between $L C M R G$ and the degree of atrophy in the cerebellar tissue. The shaded region designates the range of two standard deviations of LCMRG in the normal control group.

brainstem regions does not correlate significantly with the clinical signs of the disease (Table 6). In contrast, when the effect of the relationship between ICMRGlc and tissue atrophy is eliminated, the degree of hypometabolism still correlates significantly with the overall clinical severity. In other words, at any given level of tissue atrophy, the decline in KCMRGlc detected by PET remains significantly correlated with the clinical manifestations of OPCA (see Table 6).

\section{Discussion}

Our data demonstrate that ataxia of limb and truncal movements, along with signs of brainstem motor dysfunction, are directly related to the metabolic rate of the cerebellum and brainstem in OPCA. The findings confirm the importance of the physiological state of the cerebellum and its functional connections in the brainstem in the clinical appearance of incoordination
Table 6. Partial Correlations Between Clinical Impairment Score, lCMRGlc, and Tissue Atropby ${ }^{\mathrm{a}}$

\begin{tabular}{llc}
\hline & $\begin{array}{l}\text { ICMRGlc } \\
\text { Given } \\
\text { Tissue } \\
\text { Atrophy }\end{array}$ & $\begin{array}{l}\text { Tissue } \\
\text { Atrophy } \\
\text { Given } \\
\text { ICMRGlc }\end{array}$ \\
\hline Cerebellar vermis & $-0.42^{\mathrm{b}}$ & 0.22 \\
Left cerebellar hemisphere & $-0.44^{\mathrm{c}}$ & 0.16 \\
Right cerebellar hemisphere & $-0.33^{\mathrm{b}}$ & 0.22 \\
Brainstem & $-0.44^{\mathrm{c}}$ & -0.12 \\
\hline
\end{tabular}

When the effect of the relationhip between local cerebral metabolic rate for glucose (ICMRGlc) and tissue atrophy is eliminated, the clinical impairment score remains significantly correlated with ICMRGIc in all the regions of interest in the cerebellum and brainstem (first column). When the effect of the relationship between ICMRGlc and tissue atrophy is eliminated, the amount of tissue atrophy no longer correlates with the dinical impairment score (second column).

${ }^{\mathrm{b}} p<0.05$.

${ }^{c} p<0.01$.

of motor functions. A slightly weaker relationship was noted between the amount of atrophy visualized on CT in the cerebellar vermis and hemispheres and the severity of clinical deficit. The absence of any correlation between brainstem tissue atrophy and clinical score may relate to the difficulty in estimating the degree of brainstem atrophy in CT scans because of beam-hardening artifacts.

The scale we developed for assessing clinical severity in OPCA scores primarily ataxic components of motor impairment in OPCA. Some OPCA patients demonstrate corticospinal tract involvement as manifested by spasticity, muscle weakness, hyperreflexia, and extensor plantar responses; however, we have shown previously that metabolic rates in the cerebellum and brainstem are no different in patients with OPCA who have corticospinal tract involvement when compared to those patients with OPCA who do not display these signs [17]. Because our current study focused on ROIs within the cerebellum and brainstem, we did not include signs indicating corticospinal tract involvement in our quantitation of clinical progression in OPCA.

The correlation between ICMRGIc and tissue atrophy raises the question of whether the decrease in metabolism measured by PET can be accounted for by the amount of atrophy in the cerebellum and brainstem of patients with OPCA. That is, does the partial volume effect (decreased tissue mass and increased cerebrospinal fluid in the tissue) resulting from the loss of neurons and their synaptic connections cause the measured metabolic rate to be low? This issue cannot be settled with any certainty until a quantitative method for analyzing cerebellar and brainstem atrophy in the C'T scan is developed. Currently, we are pursuing this task. The results suggest that some of the measured decline in ICMRGlc is due directly to the effects 
of atrophy; however, Figure 2 demonstrates that some patients have no atrophy or mild atrophy and yet display severely depressed ICMRGlc. This suggests that in some patients the amount of atrophy detected by $\mathrm{CT}$ in the cerebellum and brainstem does not explain the observed hypometabolism of the tissue in these regions.

Our approach, in light of the aims of the current study, was to determine whether the clinical signs of disease are more closely linked to anatomical degeneration or to a decline in ICMRGlc. To achieve a satisfactory assessment we had to account for the tendency of these two variables to progress concomitantly. Thus, we employed the partial correlations method of analysis. The results of this analysis (see Table 6) indicate that tissue atrophy as assessed by CT is not sufficient to explain the relationship between metabolism and clinical score. In contrast, hypometabolism as determined by PET is clearly more tightly linked to the clinical severity of the disease process. This suggests that the metabolic rate of the tissue is a more important correlate of motor dysfunction in OPCA than is the degree of atrophy.

We conclude that the information provided by PET is useful in a complete assessment of OPCA in that, at any given level of tissue atrophy noted by CT, the degree of hypometabolism correlates with the clinical severity of the disease. In previous work we have found a significant decline in ICMRGlc in OPCA measurable by PET. This implies that decreased ICMRGlc measured with PET could support a clinical impression of OPCA; however, the issue of whether PET provides any clinically useful information not provided by $\mathrm{CT}$ alone remained unresolved. In our current study we have shown that PET studies may be abnormal in symptomatic patients who have minimal or no evidence of atrophy in CT scans. Thus, PET does appear to be capable of providing information that cannot be obtained from CT scans. In addition, the metabolic data that PET provides is important in assessing the severity of the disease. In further work we plan to explore the relationship between the neurological signs of disease in the various adult-onset cerebellar ataxias and the metabolic rate in specific CNS structures. Such studies will help to establish whether PET can serve as an indicator of severity in these diseases and whether differing patterns of deficit in metabolic rate can be useful in the differential diagnosis of cerebellar dysfunction

Supported in part by NIH grants NS-15655, NS-00908 and NS07222 .

We thank Dr Morton Brown for his advice concerning statistical analysis of the data and Drs B. J. Giordani and S. Berent for their assistance. We are indebted to the staff of the Division of Nuclear Medicine for their participation in this study.

\section{References}

1. Gilman S, Bloedel JR, Lechtenberg R. Disorders of the cerebellum. Philadelphia: Davis, 1981

2. Harding AE. The hereditary ataxias and related disorders. London: Churchill Livingstone, 1984

3. Eadie MJ. Olivo-ponto-cerebellar atrophy (Dejerine-Thomas type). In: Vinken PJ, Bruyn GW, eds. Handbook of clinical neurology, Vol 21. Amsterdam: North-Holland, 1975:415431

4. Eadie MJ. Olivo-ponto-cerebellar atrophy (Menzel type). In: Vinken PJ, Bruyn GW, eds. Handbook of clinical neurology, Vol 21. Amsterdam: North-Holland, 1975:433-449

5. Liversedge LA, Emery V. Electroencephalographic changes in cerebellar degenerative lesions. J Neurol Neurosurg Psychiatry 1961;24:326-330

6. Gilroy J, Lynn GE. Computerized tomography and auditory evoked potentials: use in the diagnosis of olivopontocerebellar degeneration. Arch Neurol 1978;35:143-147

7. Wadia NH, Irani P, Mehta L, Purohit A. Evidence of peripheral neuropathy in a variety of heredofamilial olivo-ponto-cerebellar degeneration frequently seen in India. In: Sobue I, ed. Spinocerebellar degenerations. Tokyo: University of Tokyo Press, 1980

8. McLeod JG, Evans W. Peripheral neuropathy in spinocerebellar degenerations. Muscle Nerve 1981;4:51-61

9. Plaitakis A, Nicklas WJ, Desnick RJ. Glutamate dehydrogenase deficiency in three patients with spinocerebellar syndrome. Ann Neurol 1980;7:297-303

10. Plaitakis A, Berl S, Yahr MD. Abnormal glutamate metabolism in an adult onset degenerative neurological disorder. Science $1982 ; 216: 193-196$

11. Plaitakis A, Berl S, Yahr MD. Neurological disorders associated with deficiency of glutamate dehydrogenase. Ann Neurol 1984; 15:144-153

12. Duvoisin RC, Chokroverty S, Lepore F, Nicklas WJ. Glutamate dehydrogenase deficiency in patients with olivopontocerebellar atrophy. Neurology 1983;33:1322-1326

13. Chokroverty S, Khedekar R, Derby B, et al. Pathology of olivopontocerebellar atrophy with glutamate dehydrogenase deficiency. Neurology 1984;34:1451-1455

14. Finocchiaro G, Taroni F, Pandolfo M, Di Donato S. Glutamate metabolism alterations in dominant OPCA. Ital J Neurol Sci 1984;(suppl 4): 134-140

15. Finocchiaro G, Taroni F, Di Donato S. Glutamate dehydrogenase in olivopontocerebellar atrophies: leukocytes, fibroblasts, and muscle mitochondria. Neurology 1986;36:550-553

16. Konagaya $Y$, Konagaya $M$, Takayanagi $T$. Glutamate dehydrogenase and its isozyme activity in olivopontocerebellar atrophy. J Neurol Sci 1986;74:231-236

17. Gilman S, Markel DS, Koeppe RA, et al. Cerebellar and brainstem hypometabolism in olivopontocerebellar atrophy detected with positron emission tomography. Ann Neurol 1988;23:223230

18. Kluin KJ, Gilman S, Markel DS, et al. Speech disorders in olivopontocerebellar atrophy correlate with positron emission tomography findings. Ann Neurol 1988;23:547-554

19. Ehrenkaufer RE, Potocki JF, Jewett DM. Simple synthesis of F18-labeled 2-fluoro-2-deoxy-D-glucose: concise communication. J Nucl Med 1984;25:333-337

20. Phelps ME, Huang SC, Hoffman EJ, et al. Tomographic measurement of local cerebral glucose metabolic rate in humans with (F-18) 2-fluoro-2-deoxy-D-glucose: validation of method. Ann Neurol 1979;6:371-388

21. Hawkins RA, Mazziotta JC, Phelps ME, et al. Cerebral glucose metabolism as a function of age in man: influence of the rate constants in the fluorodeoxyglucose method. I Cereb Blood Flow Metab 1983;3:250-253 